\title{
Zur Frage der Infusion physiologischer Kochsalzlösung.
}

Von Prof. Dr. Albert Sippel in Frankfurt a. M.

Am Vormittag des 16. Oktober v. J. wurde in meiner Privatklinik bei einer 47jährigen Frau ein kindskopfgroßer Parovarialtumor durch Laparotomie entfernt. Es war die Trennung einiger membranöser Verwachsungen nötig. Im übrigen verlief die Operation einfach. Die Ge-

1) Wiener klinische Wochenschrift 1910, No. 5. 
schwulst ließ sich stielen, und der breite Stiel wurde mit drei Ligaturen abgebunden. Am Ende der Operation wurden dem bei mir geïbten Gebrauch entsprechend 3/4 Liter einer sterilen physiologischen Kochsalzlösung vor Schluß der Bauchhöhle in diese gegossen. Drei Stunden nach dem Eingriff war die Temperatur auf 38.3 gestiegen; nachmittags 5 Uhr erreichte sie 39,5 , ging bis 8 Uhr abends auf 39,1 zurück, betrug am anderen Morgen 8 Uhr 36,8 und blieb dann normal. Entsprechend dem Temperaturverlauf stieg die Pulszahl am Nachmittag des Operationstages auf 104 und war vom nächsten Morgen ab normal. Außer dem Gefühl von Herzklopfen und Hitze bestanden während der Temperatur erhöhung keine Störungen des Allgemeinbefindens. Auffallend war nur, daß in der ersten Nacht zwei Stuhlentleerungen erfolgten. Der Wundheilungsvorgang war reaktionslos. Die Bauchdecken heilten per primam. Im Becken fand sich auch nicht die geringste Spur eines Exsudates. Der geschilderte Temperaturanstieg überraschte uns sehr. Wir sind so etwas nach derartigen Eingriffen nicht gewöhnt. Ein Infektionsvorgang war auszuschließen. Infektionen mit so rasch einsetzenden Temperaturerhöhungen sind immer hochvirulent. Sie verlaufen nicht in der kurzen Spanne Zeit, in der sich bei unserem Fall die Fieberbewegung abspielte und verlaufen auch nicht, ohne örtliche Reaktion zu machen. Wir fühıten die Störung auf Verunreinigung der infundierten Kochsalzlösung zurück. Welcher Art diese sein mochte, blieb unklar. Wir dachten auch an die neuerlich, namentlich von pädiatrischer Seite, veröffentlichen Arbeiten über Kochsalzfieber, nach denen das infundierte $\mathrm{NaCl}$ selbst fiebererregend wirken soll.

Für unsere Beobachtung verdienen nun ein besonderes Interesse gewisse Mitteilungen, welche über die intravenöse Salvarsantherapie gemacht worden sind, und besonders die kritisch zusammenfassenden Ausführungen, welche P. Ehr lich auf der Karlsruher Naturforscherversammlung erstattete. ${ }^{1}$

Von einzelnen Autoren, welche die intravenöse Anwendung des Salvarsans ausübten, war berichtet worden, daß sie danach häufig Fieber und mehr oder weniger schwere Allgemeinerscheinungen, Diarrhöen etc. auftreten sahen, während andere, welche völlig das gleiche Präparat benutzten, diese Folgezustände so gut wie niemals beobachteten. Wech selmann hat zuerst darauf aufmerksam gemacht, daß dicse Erscheinun gen nicht sowohl vom Salvarsan abhängig sein könnten, als vielmeh mit dem Bakteriengehalt des destillierten Wassers zusammenhängen müßten, das zur Herstellung der injizierten Lösung benutzt wurde. Im destillierten Wasser entwickeln sich bei längerem Aufbewahren mehr oder weniger zahlreiche Bakterien. Die Toxine dieser bei der vor dem intravenösen Gebrauch erfolgenden Sterilisation abgetöteten Bakterien sind es, welche jene Allgemeinerscheinungen hervorrufen, bei dem einen Menschen stärker, bei dem anderen weniger stark, je nach der individuellen Reaktionsfähigkeit jedes einzelnen. Bei der Anwendung eines absolut frisch destillierten Wassers, das bakterienfrei ist, traten die Nebenerscheinungen nicht mehr auf. Galewsk y (Dresden) erlebte be der Salvarsantherapie während einiger Wochen die allerschwersten All gemeinerscheinungen. Nachdem er das Wasser zu der Injektionslösung aus einer anderen Apotheke bezog, sah er von diesen Erscheinungen keine Spur mehr auftreten.

Es erübrigt sich, auf die theoretischen Erwägungen einzugehen, welche Ehrlich für die Salvarsanbehandlung an diese Feststellungen knüpfte. Für uns ist das Wesentliche die Angabe, daß der Bakteriengehalt des destillierten Wassers es ist, welcher jene Fieberbewegungen und sonstigen Allgemeinerscheinungen hervorruft. Diese Möglichkeit, so glaube ich, müssen wir in Zukunft auch bei unseren Infusionen physiologischer Kochsalzlösung in Betracht ziehen, mögen sie intravenös, intraperitoneal oder subkutan verabfolgt werden. Freilich werden die intravenösen Infusionen wegen der plötzlichen Gesamtwirkung der einverleibten Bakterienleichen die stärkste Reaktion bieten. Wir müssen stets Bedacht darauf nehmen, für solche Zwecke ein möglichst frisch hergestelltes oder in zuverlässig steriler Weise aufbewahrtes destilliertes Wasser in Anwendung zu ziehen. Natürlich läßt es sich nicht beweisen, daß in unserem Falle die Temperaturerhöhung durch den Bakteriengehalt des destillierten Wassers hervorgerufen wurde. Man wird aber zugeben müssen, daß in Anbetracht des ganzen Verlaufs die Wahrscheinlichkeit eines solchen Zusammenhangs sehr groß ist. ${ }^{2}$ )

1) Münchener medizinische Wochenschrift 1911, No. 47

${ }^{2}$ Anmerkung bei der Korrektur. Inzwischen wurde mir eine Arbeit von $\mathrm{Paul} \mathrm{Th}$. Müller in Graz bekannt (Münchener medizinische Wochenschrift 1911, No. 51), welche die Annahme einer durch den Bak. teriengehalt der physiologischen CINa-Lösung verursachten Temperatur erhöhung wesentlich stïitzt. Mïller entnahm aus 15 verschiedenen Apotheken Proben der vorhandenen Kochsalzlösung und stellte fest, daß sie sämtlich bakterienhaltig waren, und zwar schwankte der Gehalt innerhalb weiter Grenzen (68000-6050000 Keime pro cem). Demnach wer
Auch die Untersuchungen über das sogenannte Kochsalzfieber sind, soweit die in der angegebenen Richtung erforderlichen Kautelen nicht etwa schon berücksichtigt wurden, in entsprechender Weise nachzuprüfen, da möglicherweise hier Fehlerquellen liegen können, um so mehr, als der kindliche Organismus allen fiebererregenden Einwirkungen gegenüber weit stärker reagiert als der erwachsene.

Bei dieser Gelegenheit mag es mir gestattet sein, noch einmal mit einigen Worten auf eine andere Frage der Kochsalzinfusion zurückzukommen, zu der ich mich vor zwei Jahren geäußert habe. ${ }^{1}$ ) Eine experimentelle Arbeit Kawasoyes, Untersuchungen Rössles, klinische und experimentelle Beobachtungen anderer Autoren (H. Strauss u. a.) und eine eigene klinische Beobachtung hatten mich damals veranlaßt, vor der Infusion physiologischer Kochsalzlösung, wie sie allgemein bei Eklampsie geübt wurde, bei solchen Erkrankungsfällen zu warnen, welche mit Nierenschädigungen kompliziert sind. Die genannten Arbeiten hatten ergeben, daß die Zufuhr größerer Kochsalzmengen in Form der sogenannten physiologischen Lösung auf Nieren mit geschädigtem Parenchym deletär zu wirken vermochten, während gesunde Nieren die gleichen Mengen anstandslos wieder ausschieden. Inzwischen hat sich Henkel veranlaßt gesehen, dieser Frage experimentell näher zu treten. ${ }^{2}$ ) Er hat Schwangeren mit eiweißfreiem Urin vier Wochen lang täglich $10 \mathrm{~g} \mathrm{ClNa}$ per os gegeben. Der Urin blieb eiweißfrei.

Vier gesunde Schwangere erhielten an drei aufeinander folgenden Tagen je ein Liter einer 1,8 \%igen Kochsalzlösung subkutan. Der Urin blieb eiweißfrei. Vier Schwangere erhielten je einmal $5 \mathrm{ccm}$ einer $10 \%$ igen Lösung intravenös. Auch im Urin dieser Schwangeren trat danach kein Albumen auf. Eine Wöchnerin, bei der Zylinder und rote und weiße Blutkörperchen im Urin nachgewiesen wurden, erhielt $5 \mathrm{~g}$ einer $5 \%$ igen und nach zwei Tagen $5 \mathrm{~g}$ einer $10 \%$ igan Lösung intravenös, nachdem vorher das Albumen auf dem Urin geschwunden war. Es trat danach kein Eiweiß wieder auf. Bei einer Wöchnerin mit Nephritis $(1 / 2 \% 00$ Albumen) und starken Oedemen wurden $5 \mathrm{~g}$ einer $10 \%$ igen Lösung intravenös gegeben, am 26. Tag $5 \mathrm{~g}$ einer $5 \%$ igen, drei Tage später 5 g einer $10 \%$ igen Lösung. Oademe und Albumen nahmen nicht zu. Eine Frau, welche während der Gravidität schwere Nephritis und Oedeme des ganzen Körpers hatte $(8 \%$ oo Albumen), erfährt post partum aus gezeichnete Besserung. Die Oədeme gehen zurück. Die Diurese wird sehr gut. Am 19. Wochenbattstag hat sie noch $1 / 4 \%$ Albumen. Jetzt erhält sie subkutan einen Liter einer 1,8\%igen Lösung ohne Steigerung des Albumengehaltes oder Verringerung der Diurese. Auf Grund dieser Versuche glaubt $\mathrm{Henkel}$, die Bedenken gagen die subkutane Anwendung physiologischer Kochsalzlösungen bei Eklampsie mit Nephritis nicht

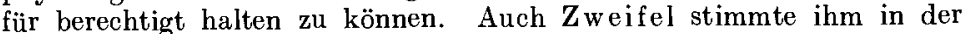
anschließenden Diskussion hierin bei.

Ich kann mich der von Henkel aus seinen Versuchen gezogenen Folgerung nicht anschließen. Soweit die Versuche an Frauen angestellt wurden, welche eiweißfreien Urin hatten, suchen sie etwas zu beweisen, ${ }^{F}$ was überhaupt nicht bestritten war. Außerdem hat wohl jeder, der bei Eklampsie Kochsalzinfusionen ausführte, die Beobachtung machen können, daß diese auch bei Nierenschädigungen nicht zum Bösen geführt haben. Es stellen demnach auch die bei den zwei leichten Albuminuriefällen angestellten Versuche im Grunde genommen nichts fest, was nicht schon bekannt war. Neu ist, daß auch eine 1,8\% hat. Das ist aber nicht viel anders, als wenn man zwei Liter einer $0,9 \%$ igen Lösung infundiert, denn in beiden Fällen werden $18 \mathrm{~g}$ CINa eingeführt. Die hypertonische Beschaffenheit der $1,8 \%$ igen Lösung wird im Blute rasch ausgeglichen.

Trotzdem es nun bekannt ist und bekannt war, daß auch Nieren, die bis zu einem gewissen Grade Parenchymschädigungen besitzen, das durch subkutane Infusion einverleibte Kochsalz schadlos wieder auszuscheiden vermögen, darf man meines Erachtens daraus doch nicht folgern, daß die Infusion größerer Mengen der physiologischen Lösung, wie man sie bei Eklampsie ausführt, für kranke Nieren etwas Gleichgültiges sei. Das

den mit $200-300 \mathrm{ccm}$ der stark keimhaltigen Lösungen mehr Bakterien infundiert, als in $5 \mathrm{ccm}$ ejner 24 stündigen Bouillonkultur des Bacterium coli oder typhi enthalten sind. Die großen Unterschiede des Keimgehalts der einzelnen Lösungen erklären, warum das eine Mal Fieber auftritt, das andere Mal nicht.

$\left.{ }^{1}\right)$ diese Wochenschrift 1910, No. 1. -- ${ }^{2}$ ) Zentralblatt für Hygiene 1911, No. 2, und Münchener medizinische Wochenschrift 1910, No. 48. 
widerlegen einwandfrei die Tierversuche Kawasoyes. Es gibt eben kranke Nieren, deren Schädigungen so gering sind, daß trotzdem eine genügende Sekretionskraft der Epithelien vorhanden ist, um mit dem Plus von Arbeitsleistung, das durch das infundierte Kochsalz ihnen zugemutet wird, ohne eigenen Nachteil fertig zu werden. Die Natur hat ja gliicklicherweise ihre physiologischen Kräfte nicht so sparsam eingerichtet, daß sie gerade nur den notwendigsten Bedürfnissen genügen, sondern ihnen einen darüber erheblich hinausgehenden Umfang gegeben. Wo aber in jedem einzelnen Fall die Grenze liegt, bis zu welcher die Funktionssteigerung der Nierenepithelien einer parenchymkranken Niere schadlos getrieben werden kann, und ebenso, wo die Grenze der Parenchymschädigung liegt, die überhaupt noch eine Funktionssteigerung verträgt, das weiß man nicht. Ich halte es daher nach wie vor für ein bedenkliches Vorgehen, bei Eklampsie mit Nephritis die subkutanen Infusionen physiologischer Kochsalzlösung weiterhin anzuwenden. Ich meine, daß man besser daran tut, statt dessen eine isotonische, d. h. 5,4\% \% ige Traubenzuckerlösung zu infundieren, wie ich seinerzeit schon in der an meinen Vortrag im Frankfurter ärztlichen Verein sich anschließenden Diskussion hervorhob. Man hat bis zu $100 \mathrm{~g}$ Traubenzucker auf einmal infundiert, ohne danach den geringsten Nachteil erlebt zu haben, und ohne Glykosurie. Die Infusion einer Zuckerlösung erfüllt neben der Einfuhr von Flüssigkeit zur Anregung der Diurese zugleich noch die Aufgabe, dem Körper eine gewisse Menge von Kalorien zuzufüh ren. 